\title{
WEAKLY CONFLUENT, 2-TO-1 MAPS ON HEREDITARILY INDECOMPOSABLE CONTINUA
}

\author{
JO W. HEATH
}

(Communicated by James E. West)

\begin{abstract}
One of the most important open questions today in the study of exactly 2-to-1 maps is whether or not such a map can be defined on the pseudoarc. We show that there is no weakly confluent 2-to-1 map defined on the pseudoarc. More precisely, it is shown that any reduced weakly confluent 2-to1 map defined on a hereditarily indecomposable metric continuum must be a confluent local homeomorphism. It follows from this that if there is a weakly confluent 2-to-1 map from a hereditarily indecomposable continuum $X$ onto a continuum $Y$, then neither $X$ nor $Y$ can be treelike.
\end{abstract}

\section{INTRODUCTION}

The first example of a hereditarily indecomposable continuum was a pseudoarc constructed by Knaster [7]. He constructed the continuum by carefully removing open sets from a disk in the plane leaving a continuum symmetric about the origin. By identifying each point in Knaster's pseudoarc with its symmetric image, a map is defined that is exactly 2-to-1 except at one point, the origin. A natural question, first asked by J. Mioduszewski in the early 1960s, is whether a map that is exactly 2-to-1 (at every point) can be defined on the pseudoarc.

Ideas from $[6,10]$ are compiled in Lemma 1 to show that confluence together with 2-to-1 is a powerful combination. Specifically, if $f$ is a confluent 2-to-1 map defined on a continuum then there is a nondegenerate subcontinuum $X$ of the domain such that $f$ restricted to $X$ is a 2-to-1 local homeomorphism, $X$ does not have the fixed point property, and neither $X$ nor $f(X)$ is treelike. In particular, no such map can be defined on the pseudoarc. As a partial converse to Lemma 1, each local homeomorphism is $k$-to- 1 and confluent [13, pp. 148, 200]; but, of course, the $k$ need not be 2 .

In [1] Davis shows that each semiconfluent map defined on a hereditarily indecomposable continuum is confluent. An immediate corollary then is that there is no 2-to-1 semiconfluent map defined on a pseudoarc. We show in Corollary 1 that there is in fact no weakly confluent 2-to-1 map defined on the pseudoarc, a stronger corollary since Maćkowiak has shown [9, p.254] that

Received by the editors June 11, 1991.

1991 Mathematics Subject Classification. Primary 54C10.

Key words and phrases. Weakly confluent, 2-to-1 map. 
semiconfluence implies weak confluence. Corollary 1 follows from our main result, Theorem 1, that each reduced, weakly confluent, 2-to-1 map defined on a hereditarily indecomposable continuum is confluent.

In contrast to these results concerning the pseudoarc, there is a 2-fold covering map (and hence a 2-to-1 confluent map) from the pseudocircle onto itself. (The details of this construction are in the second section.) A pseudocircle is any circularly chainable, hereditarily indecomposable, separating plane continuum. And, like the pseudoarc, each of its proper subcontinua are pseudoarcs.

A glossary of definitions of terms used in this paper are in the last section. All spaces are assumed metric.

\section{LEMMA, THEOREM, AND COROLLARIES}

If $f$ is a 2-to-1 map on the pseudoarc, and if $X$ is a subcontinuum of the domain minimal with respect to $X=f^{-1}(f(X))$, then $f$ restricted to $X$ is still a 2-to-1 map on a pseudoarc and it is now reduced; that is, if $C$ is a proper subcontinuum of the image then its preimage is not connected. So, if there is a 2-to-1 map on the pseudoarc, there is a reduced one. Likewise, if there is a 2-to-1 map on a treelike and/or hereditarily indecomposable continuum, then there is a reduced one. Furthermore, since the restriction is to a continuum that is the complete preimage of a continuum, the reduced function is confluent or weakly confluent if the original function is confluent or weakly confluent. Thus the hypothesis that the map be reduced is not restrictive in these settings.

Lemma 1. Suppose $f$ is a reduced, confluent, 2-to-1 map from a continuum $X$ onto $Y$. Then

(1) $f$ is a local homeomorphism;

(2) $X$ does not have the fixed point property;

(3) neither $X$ nor $Y$ is treelike; and

(4) $X$ is hereditarily indecomposable iff $Y$ is hereditarily indecomposable.

Proof. In [6] a crisp map was defined to be one such that each continuum $C$ in the image has exactly two components in its preimage, and the function maps each homeomorphically onto $C$. Thus the property that $f$ is crisp is equivalent to our hypothesis that $f$ is reduced, confluent, and 2-to-1. In [6] it is shown that each crisp map is a local homeomorphism (Theorem 1), that $X$ does not have the fixed point property (Corollary 1), and that $Y$ is not treelike (Theorem 2 ). The fact that $X$ cannot be treelike follows from McLean's result [10] that the confluent image of a treelike continuum is treelike. Part 4 of this lemma is Corollary 3 in [6].

Theorem 1. Suppose that $f$ is a reduced, 2-to-1, weakly confluent map from a hereditarily indecomposable continuum $X$ onto $Y$. Then

(1) $f$ is confluent;

(2) $f$ is a local homeomorphism;

(3) $X$ does not have the fixed point property;

(4) neither $X$ or $Y$ is treelike; and

(5) $Y$ is hereditarily indecomposable.

Proof. Because of Lemma 1 , all we need to show is that $f$ is confluent. It was shown in [5] that $f$ is crisp (and hence confluent) if $f$ is reduced, $X$ is 


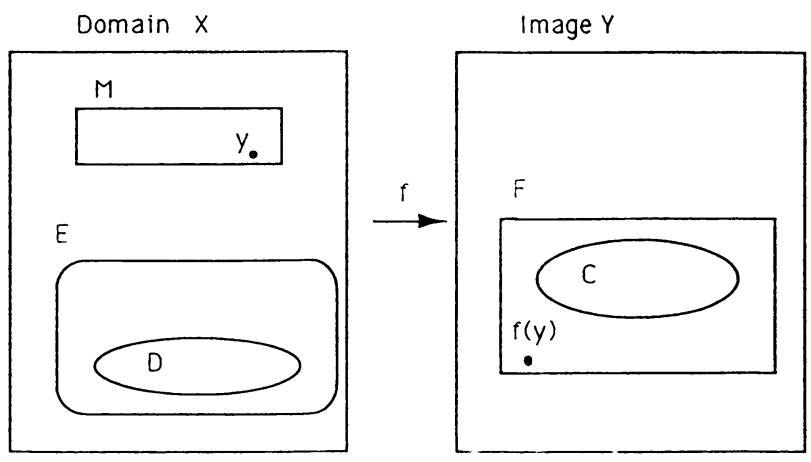

FIGURE 1

hereditarily indecomposable, and each connected nondense subset of the image has connected preimage. So we will assume on the contrary that there is a connected nondense subset $C$ of $Y$ such that $D=f^{-1}(C)$ is connected.

First note that the function $f$ restricted to $f^{-1}(\bar{C})$ is a 2-to-1 map defined on a compactum. Since $f$ is continuous, $f$ maps the continuum $\bar{D}$ onto $\bar{C}$ so, by the "Interior Lemma" in [4], there is a nonempty open (relative to $f^{-1}(\bar{C})$ ) set $U$ in $\bar{D}$. Since $D$ is dense in $\bar{D}$, there is a point $x$ of $D$ in $U$. This means that $x$ is not a limit point of $f^{-1}(\bar{C}) \backslash \bar{D}$ and, since $D$ is the complete preimage of $C$, the twin point (see glossary) $\hat{x}$ is also in $D$.

Since $f$ is reduced, $f^{-1}(\bar{C})$ is not connected, so there is a component $M^{\prime}$ of $f^{-1}(\bar{C})$ other than the one containing $\bar{D}$. The component $M^{\prime}$ can be enlarged slightly to a continuum $M$ that misses $\bar{D}$ and contains a point $y$ that does not map to $\bar{C}$. Let $F$ denote the continuum $f(M \cup \bar{D})$ containing both $\bar{C}$ and $f(y)$. Since $f$ is weakly confluent, some component $E$ of $f^{-1}(F)$ maps onto $F$. This component contains $D$ since $D$ contains the complete inverse of $C$ and is connected, and it contains $\bar{D}$ properly since some point of $E$ maps to $f(y)$. (See Figure 1.)

Since $X$ is hereditarily indecomposable, the point $x$ must be a limit point of $E \backslash f^{-1}(\bar{C})$ since no proper subcontinuum of $E$ can have interior in $E$ and since $x$ is not a limit point of $f^{-1}(\bar{C}) \backslash \bar{D}$. Thus $x$ is a limit of a sequence $\left\{x_{i}\right\}$ of points whose twins are in $M$. But these twins must converge to $x$ or to $\hat{x}$ by the semicontinuity [11] of the twin function. This is a contradiction since neither $x$ nor $\hat{x}$ is in the continuum $M$.

Corollary 1. There is no weakly confluent 2-to-1 map defined on the pseudoarc or on any hereditarily indecomposable treelike continuum.

Proof. This result follows directly from Theorem 1 and the earlier discussion that each weakly confluent 2-to-1 map defined on any hereditarily indecomposable treelike continuum has a reduced restriction to subcontinuum that is still weakly confluent and 2-to-1.

In 1972, Lelek (see [8]) introduced the notion of class $W$ : a continuum $C$ is in class $W$ if every map onto $C$ is weakly confluent. Thus from Corollary 1 we infer that no $W$-space can be the 2-to-1 image of the pseudoarc or of any hereditarily indecomposable treelike continuum. Davis has shown [2] that each atriodic continuum $C$ such that $\check{H}^{1}(C ; Z)=0$ is in class $W$; thus any 
acyclic atriodic continuum is in class $W$. Earlier, Grispolakis and Tymchatyn [3] showed that every treelike atriodic continuum is in class $W$.

Corollary 2. There is no 2-to-1 map from the pseudoarc or any hereditarily indecomposable treelike continuum onto any continuum in class $W$.

\section{EXAMPLE}

Using ideas lifted from Rogers [12], we construct a 2-to-1 confluent map from the pseudocircle onto itself.

Example 1. The pseudocircle admits a 2-fold cover onto itself.

Construction. Let $Y$ denote a pseudocircle. Since $Y$ is a separating plane continuum, we can embed $Y$ in the annulus $A=\{(r, \theta): 1 \leq r \leq 2\}$, using polar coordinates, in such a way that $Y$ separates the inner circle $\{r=1\}$ from the outer circle $\{r=2\}$. Now let $p$ denote the usual 2-fold cover from $A$ onto $A$ defined by $p(r, \theta)=(r, 2 \theta)$. Since $p$ is confluent, $p^{-1}(Y)$ has either one or two components; we will suppose that it has two components $K$ and $L$.

If neither $K$ nor $L$ separates the inner circle from the outer, then there is an $\operatorname{arc} \alpha$ in $A$ from the inner to the outer circle that misses $K \cup L$, and so its image, $p(\alpha)$, is a continuum in $A$ from the inner to the outer circle that misses $Y$. So we may assume that $K$ separates the two circles. The other component $L$ is either inside $K$ (i.e., not in the component of $A \backslash K$ containing $\{r=2\}$ ) or outside; we assume the former. Let $\left(r_{1}, \theta_{1}\right)$ denote a point of $K$ with maximum distance from the origin. Since both $K$ and $L$ map onto $Y$, the symmetric point $\left(r_{1}, \theta_{1}+\pi\right)$ belongs to $L$. To complete the contradiction we infer that, since $L$ is inside $K$, the radial arc from $\left(r_{1}, \theta_{1}+\pi\right)$ to the outer circle intersects $K$ in a point that is further away from the origin than $\left(r_{1}, \theta_{1}\right)$. This establishes the fact that $X=p^{-1}(Y)$ is a continuum.

Now we need to see that $X$ is a pseudocircle. Since $X$ separates the inner circle from the outer in $A, X$ is a separating plane continuum. Using the fact that each proper subcontinuum of $Y$ is a pseudoarc and Lemma 1, we see that $p$ restricted to $X$ is a reduced confluent map; we infer, again from Lemma 1, that $X$ is hereditarily indecomposable since $Y$ is. Furthermore, $X$ is circularly chainable since any circular chain covering $Y$ consisting of fundamental open sets lifts to a circular chain covering $X$.

\section{Definitions}

1. Confluent. A function is confluent if for each continuum $C$ in the image, each component of the preimage of $C$ maps onto $C$.

2. Continuum. A topological space is a continuum if it is connected, compact, and metric.

3. Crisp. A map $f$ is crisp if for each continuum $C$ in the image there are exactly two components of the preimage of $C$ and $f$ maps each of these components homeomorphically onto $C$.

4. Local homeomorphism. A function $f$ is a local homeomorphism if for each point $p$ in the domain there is an open set $U$ containing $p$ such that $f$ is a homeomorphism on $U$ and $f(U)$ is open.

5. Map. A function is a map if it is continuous. 
6. Pseudocircle. A circularly chainable, hereditarily indecomposable, separating plane continuum.

7. Reduced. A function is reduced if no proper subcontinuum of the image has a connected preimage.

8. Semiconfluence. A map is semiconfluent if, for each two components $C_{1}$ and $C_{2}$ of the preimage of a continuum $C$ in the image space, either $f\left(C_{1}\right) \subseteq f\left(C_{2}\right)$ or $f\left(C_{2}\right) \subseteq f\left(C_{1}\right)$.

9. Semicontinuous (Mioduszewski [11]). An involution $f$ defined on a compact space is semicontinuous if whenever $\left\{x_{i}\right\}$ converges to $x$ and $\left\{f\left(x_{i}\right)\right\}$ converges to $y$, then $y$ is either $x$ or $f(x)$.

10. Twin $(\hat{p})$. For each point $p$ in the domain of a 2-to-1 map $f$, let $\hat{p}$, called the twin of $p$, denote the other point of the domain of $f$ that maps the same as $p$ under $f$. Analogously, define $\hat{D}=\{\hat{x}: x \in D\}$, for any subset $D$ of the domain of $f$.

11. 2-to-1. A function is 2-to-1 if the preimage of each point in the image has exactly two points.

12. Weak confluence. A function is weakly confluent if for each continuum $C$ in the image, at least one component of the preimage of $C$ maps onto $C$.

\section{REFERENCES}

1. J. F. Davis, Semi-confluent mapping on continua, Proceedings of the Guilford College Sesquicentennial Topology Conference 1988, Guilford College, Greensboro, NC, 1988.

2. __ Atriodic acyclic continua and class W, Proc. Amer. Math. Soc. 90 (1984), 477-482.

3. J. Grispolakis and E. D. Tymchatyn, Continua which are images of weakly confluent mappings only. II, Houston J. Math. 6 (1980), 375-387.

4. Jo Heath, The structure of (exactly) 2-to-1 maps on metric continua, Proc. Amer. Math. Soc. 110 (1990), 549.

5. _ 2-to-1 maps on hereditarily indecomposable continua, Trans. Amer. Math. Soc. 328 (1991), 433-444.

6. _ 2-to-1 maps with hereditarily indecomposable images, Proc. Amer. Math. Soc. 113 (1991), 839-846.

7. B. Knaster, Un continu dont tout sous-continu est indécomposable, Fund. Math. 3 (1922), 247-286.

8. S. Nadler, Jr., Hyperspaces of sets, Marcel Dekker, New York, 1978, p. 497.

9. T. Maćkowiak, Semiconfluent mappings and their invariants, Fund. Math. 79 (1973), 251264.

10. T. Bruce McLean, Confluent images of tree-like curves are tree-like, Duke Math. J. 39 (1972), 465-473.

11. J. Mioduszewski, On two-to-one continuous functions, Dissertationes Math. (Rozprawy Mat.) 24 (1961), 42.

12. J. T. Rogers, Jr., Homogeneous, separating plane continua are decomposable, Michigan Math. J. 28 (1981), 317.

13. G. T. Whyburn, Analytic topology, Amer. Math. Soc., Providence, RI, 1942.

Department of Mathematics, Auburn University, Auburn University, Alabama 35649 5310

E-mail address: HEATH@DUCVAX.AUBURN.EDU 